\title{
Vape-Associated Pulmonary Injury (VAPI) Presenting With a "Miliary" Pattern on Imaging
}

\author{
Marcus A. Threadcraft ${ }^{1}$, Robert Case Jr. ${ }^{2}$ \\ 1. Internal Medicine, University of Florida College of Medicine, Gainesville, USA 2. Internal Medicine, University of \\ Florida Health, Gainesville, USA
}

Corresponding author: Marcus A. Threadcraft, m.threadcraft@ufl.edu

\begin{abstract}
Electronic (E)-cigarette use or vaping is associated with pulmonary injury. Users can present with wideranging symptoms, varying degrees of pulmonary injury, and respiratory distress. Lung injury secondary to vaping is associated with a variety of patterns on pulmonary imaging. Typical radiographic findings are consistent with bilateral, basilar ground-glass opacities and or consolidation with septal thickening. We present a case of vape-associated pulmonary injury (VAPI) in a previously healthy adult who was found to have atypical radiographic findings. A 34-year-old male presented with a chief complaint of a two-week history of malaise, nausea, cough, and worsening shortness of breath. A chest CT scan without contrast revealed diffuse nodules in a miliary pattern. The patient reported a six-month history of tetrahydrocannabinol (THC) vape use. Bronchoscopy with cytologic analysis confirmed findings consistent with the VAPI. To our knowledge, this is the first report of a "miliary" pattern of infiltrates and nodules in a patient with VAPI. This pattern on CT imaging led to increased suspicion for other possible etiologies, including tuberculosis. Thus, moving forward, we believe that VAPI needs to be considered in the differential diagnosis if a patient presents with radiographic findings consistent with a miliary or diffuse micronodular pattern.
\end{abstract}

Review began 02/02/2021 Review ended 02/11/2021 Published 02/16/2021

\section{Copyright 2021}

Threadcraft et al. This is an open access article distributed under the terms of the Creative Commons Attribution License CC-BY 4.0., which permits unrestricted use, distribution, and reproduction in any medium, provided the original author and source are credited.
Categories: Internal Medicine, Radiology, Pulmonology

Keywords: vape, e-cigarette, miliary, micronodular, pulmonary injury, thc, vapi

\section{Introduction}

As an alternative to traditional cigarette smoking, electronic (E)-cigarettes or vaping has become popular among young adults and teens $[1,2]$. Through the use of small handheld devices that heat and aerosolize liquids into ultrafine particles, vape users inhale nicotine and other noxious compounds deep into the lungs $[3,4]$. Since 2019, there have been increasing reports of vape-induced pulmonary disease with some severe enough to cause death [3]. Of note, vape cartridges containing tetrahydrocannabinol (THC) oil, the psychoactive, "high" producing compound found in marijuana, is frequently used by E-cigarette users [3]. THC-containing E-cigarettes were linked to lung injury through laboratory data findings of Vitamin E acetate [5]. As an additive in THC vape cartridges, vitamin E acetate was present in the bronchoalveolar lavage (BAL) fluid of patients with vape associated pulmonary injury (VAPI) [5].

VAPI is a relatively new diagnosis and was the subject of intense investigation by the Centers for Disease Control and Prevention (CDC) in the Fall of 2019. A diagnosis of exclusion, VAPI, tends to present as an acute or subacute systemic deterioration in young, healthy patients with few known comorbidities [6]. General and non-specific constitutional symptoms may be present, but symptoms can range in presentation and severity. Roughly $97 \%$ of patients present with respiratory symptoms such as cough, dyspnea, and chest pain, with $77 \%$ also presenting with gastrointestinal symptoms [7]. We report the case of an otherwise healthy young man who presented with malaise, worsening shortness of breath, and anorexia who was found on chest CT to have innumerable pulmonary nodules in a "miliary" pattern.

\section{Case Presentation}

A 34-year-old male with no relevant past medical history presented with a two-week history of malaise, fatigue, cough, worsening shortness of breath, and 25-pound weight loss secondary to nausea and diarrhea. He denied any pleuritic chest pain, chest tightness, sputum production, or hemoptysis. One week prior, he was seen by another healthcare provider and diagnosed with bronchitis after undergoing a chest X-ray. He reported completing a three-day course of once-daily azithromycin $500 \mathrm{mg}$ tablets, which provided no symptom relief. He denied body aches but reported a "weird" feeling in his chest. Upon further questioning, he denied any recent international travel, recent sick contacts, or high-risk behaviors. He reported working a corporate job and had not traveled more than 10 miles from his home each day for the past three months.

In the emergency department, he was ill-appearing, tachycardiac, and tachypneic with diminished breath sounds toward the lower lobes bilaterally. Oxygen saturations ranged from $89 \%-95 \%$ on room air. He was placed on two to six liters of oxygen delivered via nasal cannula, and oxygen saturations improved to greater than $96 \%$ at rest. Initial laboratory workup was significant for C-reactive protein of $386 \mathrm{mg} / \mathrm{L}$, AST of 


\section{Cureus}

$101 \mathrm{IU} / \mathrm{L}$, ALT of $86 \mathrm{IU} / \mathrm{L}$, white blood cell count of 10.7 thou/cu mm (neutrophil predominant) with a platelet count of 478 thou/cu mm, and an INR of 1.8. A chest X-ray was significant for increased interstitial markings with a few air bronchograms suggesting right lower lobe focal consolidation (Figures 1,2). A subsequent non-contrast chest CT scan showed numerous miliary pulmonary nodules in a random pattern with bibasilar patchy consolidations (Figure 3). Given the increased suspicion for miliary tuberculosis, the patient was placed in isolation and under respiratory precautions. Infectious disease and pulmonology were consulted to assist in further workup and management.

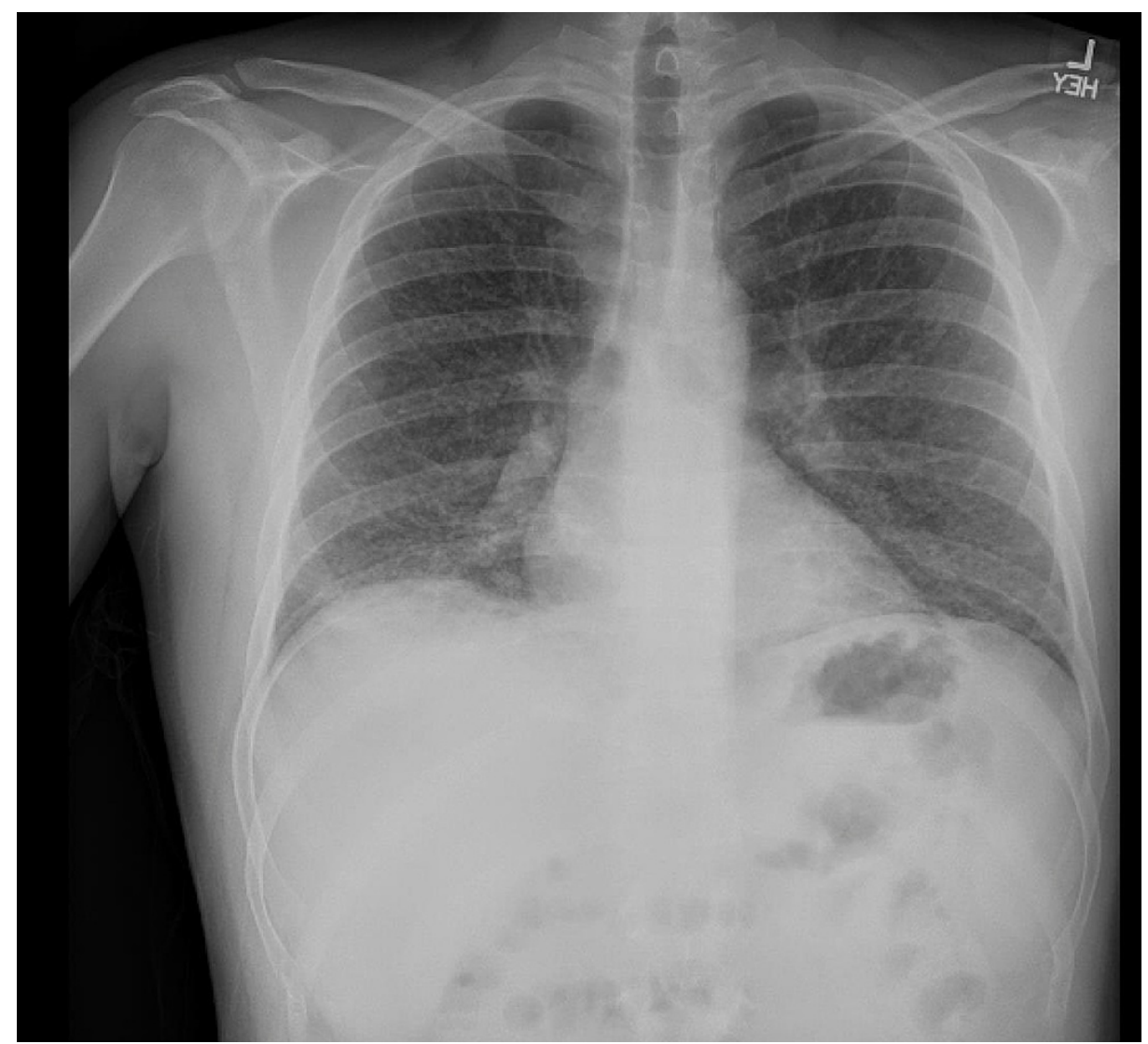

FIGURE 1: Chest X-ray in the emergency department.

Image shows increased interstitial markings with suggestion of a right lower lobe more focal consolidation. 


\section{Cureus}

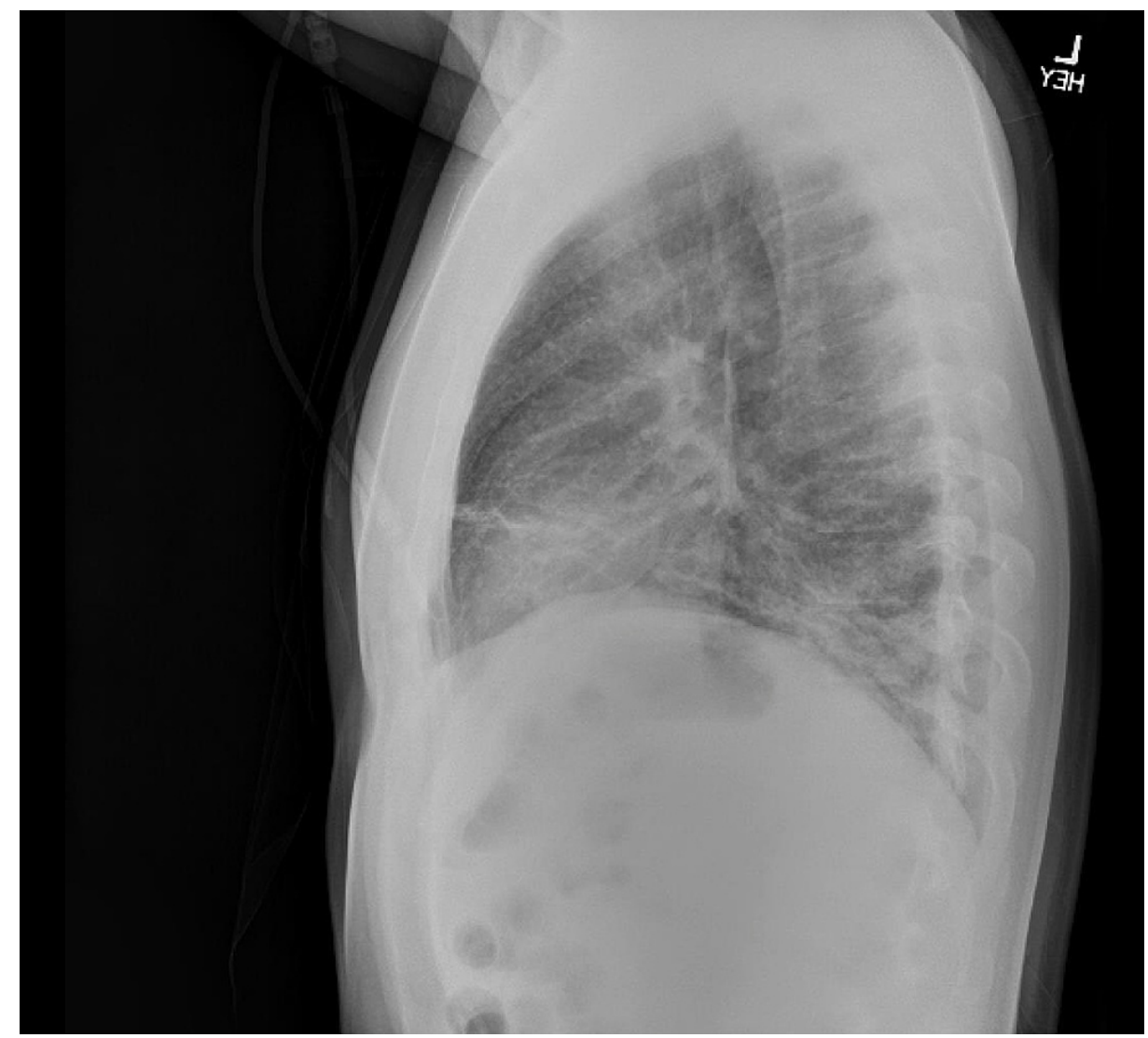

FIGURE 2: Chest X-ray in the emergency department (lateral view).

Image shows increased interstitial markings with suggestion of a right lower lobe more focal consolidation.

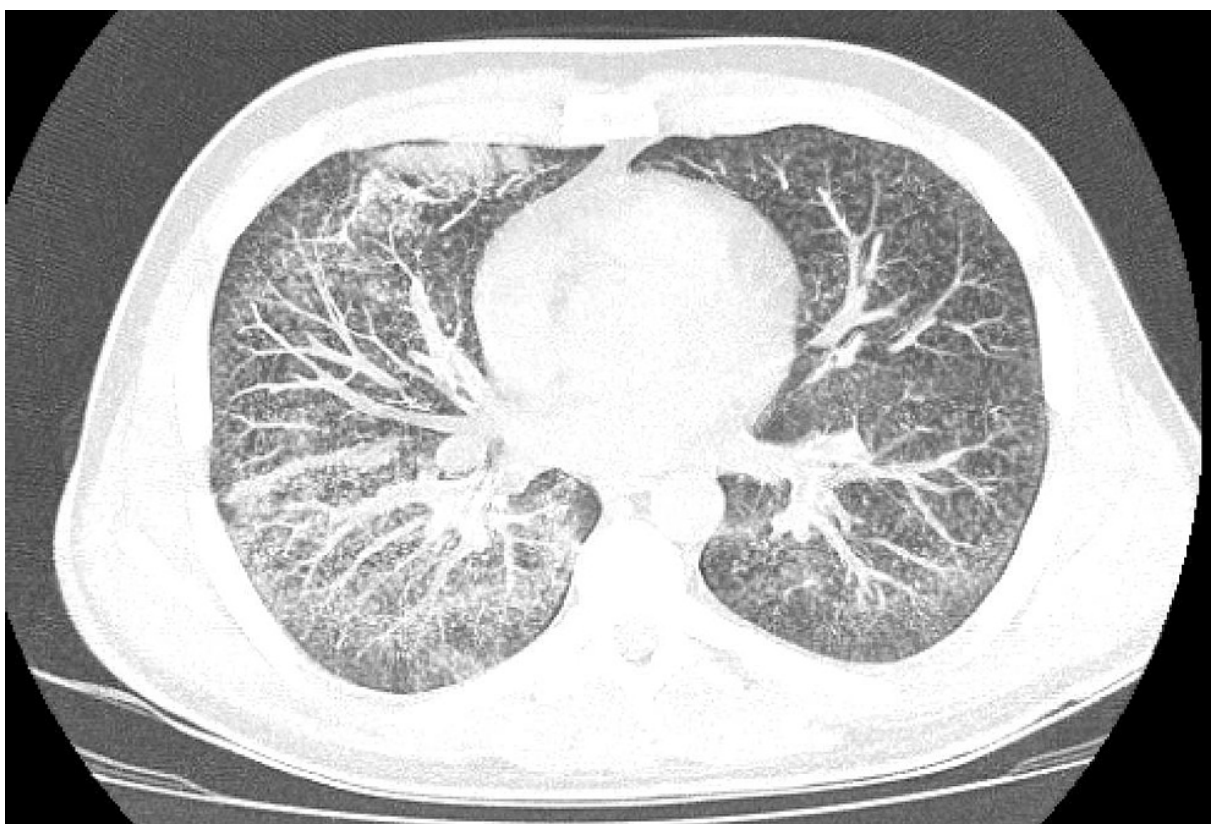

FIGURE 3: Chest CT on admission.

Image shows scattered pulmonary micronodules seen in a predominantly random miliary pattern with bibasilar patchy opacities.

Further testing, including respiratory and GI polymerase chain reaction (PCR) panels, HIV, hepatitis panel, 
QuantiFERON, and blood cultures, were unrevealing. As malignancy with pulmonary metastasis was also a diagnostic consideration, tumor markers and a non-contrast CT of the abdomen and pelvis were ordered. The CT scans were unrevealing, and all tumor markers were within normal limits. During his hospitalization, the patient revealed his history of THC-vape use for the preceding six months. This new information heightened our clinical suspicion for VAPI, and a flexible bronchoscopy with biopsy was pursued to confirm the diagnosis and rule out other infectious etiologies. Testing for Histoplasma, tularemia, and cryptococcus were performed and returned negative results. Histopathology from the bronchoscopy revealed intraalveolar granulation tissue consistent with organizing pneumonia, with no evidence of acid-fast organisms, pneumocystis, fungi, or malignancy. Cytology later confirmed the presence of acute and chronic inflammation with lipid-laden macrophages. Systemic corticosteroids were initiated after identifying lipoid pneumonia in conjunction with vape use. Within three days of admission, his transaminitis began to improve, along with his respiratory function with a decreasing need for oxygen support. After five days, the patient was discharged and instructed to follow-up in the pulmonary clinic within a week and advised to discontinue vaping.

\section{Discussion}

To our knowledge, this is the first example of a "miliary" pattern on chest imaging associated with vaping. Before confirming the diagnosis, infectious etiologies such as tuberculosis were much higher on the differential diagnosis, but the patient had no known exposures or risk factors. VAPI has been associated with several radiographic patterns such as diffuse alveolar damage, organizing pneumonia, and hypersensitivity pneumonitis patterns [8]. CT imaging often demonstrates areas of consolidation, ground-glass changes, nodular consolidation, septal thickening, and mosaic attenuation [9]. The diffuse micronodules in our case were initially concerning for tuberculosis or malignancy. The diagnosis of VAPI and lipoid pneumonia were arrived at after carefully ruling out alternative etiologies and confirming the presence of lipid-laden macrophages. His improvement with corticosteroids and the cessation of THC-vape use also support the diagnosis of VAPI in this case. As our clinical understanding of this condition continues to evolve, our case provides value in demonstrating another radiographic pattern associated with the diagnosis.

The mechanism of lung injury in VAPI is still under investigation, but studies have shown that damage to the lung parenchyma is likely linked to a chemical pneumonitis [10]. The aerosolized liquids inhaled via Ecigarettes contain an array of chemical compounds. Nicotine based E-cigarettes, in addition to nicotine, may include propylene glycol, glycerin, polycyclic aromatic hydrocarbons, nitrosamines, volatile organic chemicals, toxic metals, flavoring compounds, and endotoxins [11,12]. Additionally, E-cigarettes can deliver recreational drugs, including THC-based oils, to users via vape cartridges. In this patient's case, use of a vape cartridge with a reported 90\% THC-based oil. The Food and Drug Administration (FDA) has identified and demonstrated that many THC-containing vape products also contain vitamin E acetate [5]. The degree to which vitamin $\mathrm{E}$ acetate is the direct cause of lung injury in VAPI is yet to be determined but is believed to be associated with acute lung injury. The CDC have recommended that THC-containing vape products, especially those containing vitamin $\mathrm{E}$ acetate, be avoided [5].

\section{Conclusions}

VAPI should be considered in a patient with a history of E-cigarette use, who presents with pulmonary symptoms and bilateral infiltrates on imaging. CT imaging may be useful in highlighting specific patterns of inflammation that are associated with VAPI. Our case adds a micronodular or "miliary" pattern of nodules to the list of potential radiographic patterns associated with this condition. The patient in our case had a brisk recovery with corticosteroids and cessation of vaping.

\section{Additional Information}

\section{Disclosures}

Human subjects: All authors have confirmed that this study did not involve human participants or tissue. Conflicts of interest: In compliance with the ICMJE uniform disclosure form, all authors declare the following: Payment/services info: All authors have declared that no financial support was received from any organization for the submitted work. Financial relationships: All authors have declared that they have no financial relationships at present or within the previous three years with any organizations that might have an interest in the submitted work. Other relationships: All authors have declared that there are no other relationships or activities that could appear to have influenced the submitted work.

\section{References}

1. Dinardo P, Rome ES: Vaping: the new wave of nicotine addiction. Cleve Clin J Med. 2019, 86:789-798. 10.3949/ccjm.86a.19118

2. Know the Risks: E-cigarettes \& Young People | U.S. Surgeon General's Report . (2018). Accessed: November 24, 2020: http://cigarettes.surgeongeneral.gov/.

3. CDC: Outbreak of Lung Injury Associated with the Use of E-Cigarette, or Vaping, Products . (2020). Accessed: December 1, 2020: https://www.cdc.gov/tobacco/basic_information/e-cigarettes/severe-lungdisease.html.

4. NIDA. Vaping Devices (Electronic Cigarettes) Drug Facts. (2020). Accessed: November 27, 2020: 


\section{Cureus}

https://www.drugabuse.gov/publications/drugfacts/vaping-devices-electronic-cigarettes.

5. Blount BC, Karwowski MP, Shields PG, et al.: Vitamin E acetate in bronchoalveolar-lavage fluid associated with EVALI. N Engl J Med. 2020, 382:697-705. 10.1056/nejmoa1916433

6. EMCrit: Vaping Associated Pulmonary Injury (VAPI) . (2020). Accessed: November 23, 2020: https://emcrit.org/ibcc/vaping-associated-pulmonary-injury/.

7. Layden JE, Ghinai I, Pray I, et al.: Pulmonary illness related to E-cigarette use in Illinois and Wisconsin Final Report. N Engl J Med. 2020, 382:903-916. 10.1056/nejmoa1911614

8. Henry TS, Kanne JP, Kligerman SJ: Imaging of vaping-associated lung disease . N Engl J Med. 2019, 381:14861487. 10.1056/NEJMc1911995.

9. Hentry TS, Kligerman SJ, Raptis CA, et al.: Imaging findings of vaping-associated lung injury . Am J Roentgenol. 2020, 214:498-505. 10.2214/AJR.19.22251

10. Fonseca Fuentes X, Kashyap R, Hays JT, et al.: VpALI-vaping-related acute lung injury: a new killer around the block. Mayo Clin Proc. 2019, 94:2534-2545. 10.1016/j.mayocp.2019.10.010

11. Burstyn I: Peering through the mist: systematic review of what the chemistry of contaminants in electronic cigarettes tells us about health risks. BMC Public Health. 2014, 14:18. 10.1186/1471-2458-14-18

12. Pisinger C, Dossing M: A systematic review of health effects of electronic cigarettes . Prev Med. 2014, 69:248-260. 10.1016/j.ypmed.2014.10.009 